\title{
Terminalia chebula extract prevents scopolamine-induced amnesia via cholinergic modulation and anti-oxidative effects in mice
}

\author{
Min-Soo Kim ${ }^{1,2}$, Dong Young Lee ${ }^{3}$, Jun Lee ${ }^{1,2}$, Hyun Woo Kim³ , Sang Hyun Sung ${ }^{3}$, Jung-Soo Han ${ }^{4}$
} and Won Kyung Jeon ${ }^{1,2^{*}}$

\begin{abstract}
Background: Terminalia chebula Retz. (Combretaceae) is a traditional herbal medicine that is widely used in the treatment of diabetes, immunodeficiency diseases, and stomach ulcer in Asia. However, the anti-amnesic effect of $T$. chebula has not yet been investigated. The present study was designed to determine whether T. chebula extract (TCE) alleviates amnesia induced by scopolamine in mice. We also investigated possible mechanisms associated with cholinergic system and anti-oxidant effects.
\end{abstract}

Methods: TCE (100 or $200 \mathrm{mg} / \mathrm{kg}$ ) was orally administered to mice for fourteen days (days 1-14), and scopolamine was intraperitoneally injected to induce memory impairment for seven days (days 8-14). Learning and memory status were evaluated using the Morris water maze. Hippocampal levels of acetylcholine (ACh), acetylcholinesterase (AChE) and choline acetyltransferase (ChAT) were measured ex vivo. Levels of reactive oxygen species (ROS), nitric oxide (NO), and malondialdehyde (MDA) in the hippocampus were also examined.

Results: In the Morris water maze task, TCE treatment reversed scopolamine-induced learning and memory deficits in acquisition and retention. TCE reduced hippocampal AChE activities and increased ChAT and ACh levels in the scopolamine-induced model. Moreover, TCE treatment suppressed scopolamine-induced oxidative damage by ameliorating the increased levels of ROS, NO, and MDA.

Conclusion: These findings suggest that TCE exerts potent anti-amnesic effects via cholinergic modulation and antioxidant activity, thus providing evidence for its potential as a cognitive enhancer for amnesia.

Keywords: Terminalia chebula, Amnesia, Cholinergic system, Oxidative damage

\section{Background}

As many countries are transforming into aging societies, an increasing number of individuals in elderly populations suffers from memory loss and amnesia: worldwide cost of care and medicine for amnesia was reported to be $\$ 604$ billion in 2010 and has been increasing annually

\footnotetext{
* Correspondence: wkjeon@kiom.re.kr

${ }^{1}$ Herbal Medicine Research Division, Korea Institute of Oriental Medicine, Daejeon 34054, South Korea

${ }^{2}$ Convergence Research Center for Diagnosis, Treatment and Care System of Dementia, Korea Institute of Science and Technology, Seoul 02792, South Korea

Full list of author information is available at the end of the article
}

[1]. The prevention or treatment of amnesia is thus an urgent issue to address.

The hippocampus and cortex are heavily involved in maintaining and regulating memory; changes in levels of the neurotransmitter acetylcholine (ACh), which is released via cholinergic projections to these areas from the basal forebrain, have been reported to affect cognitive function and have been implicated in memory loss [2]. Therapeutic interventions aiming to alleviate cognitive impairments have thus targeted $\mathrm{ACh}$ regulation and degradation; acetylcholinesterase (AChE) inhibitors blocking ACh hydrolysis have been administered to treat amnesia [3] and are widely prescribed to alleviate general

(c) The Author(s). 2018 Open Access This article is distributed under the terms of the Creative Commons Attribution 4.0 International License (http://creativecommons.org/licenses/by/4.0/), which permits unrestricted use, distribution, and reproduction in any medium, provided you give appropriate credit to the original author(s) and the source, provide a link to the Creative Commons license, and indicate if changes were made. The Creative Commons Public Domain Dedication waiver (http://creativecommons.org/publicdomain/zero/1.0/) applies to the data made available in this article, unless otherwise stated. 
cognitive decline [4]. However, these drugs have short half-lives and adverse reactions, including hepatotoxicity and nausea [5]. By contrast, medicinal herbs and plants exhibit fewer side effects and drug interactions [6]; identifying effective alternative medicines could thus yield valuable contributions to the treatment of amnesia.

Terminalia chebula Retz. (Combretaceae) has been used as a traditional medicine to treat diabetes, immunodeficiency diseases, and stomach ulcers in across Asia [7-9]. These medicinal extracts contain terpenes, flavonoids, and alkaloids, all of which exhibit therapeutic efficacy [10]. Several in vitro studies have further reported that Terminalia chebula extract (TCE) has antiAChE and anti-oxidative effects: TCE has been shown to inhibit $\mathrm{H}_{2} \mathrm{O}_{2}$-induced $\mathrm{PC} 12$ cell death [11], and its methanolic and ethyl acetate fractions have demon strated varying degrees of AChE inhibitory activity [12, 13]. Despite the progress made to explore the therapeutic properties of TCE, whether the extract features an antiamnesic effect has not yet been investigated.

Scopolamine is a muscarinic ACh receptor antagonist that can cause learning and memory deficits by disrupting cholinergic neurotransmission; this compound has been used to induce amnesia in experimental murine models [14]. In the present study, we evaluated the memory-enhancing effects of TCE on scopolaminetreated mice using the Morris water maze test. We subsequently investigated the levels of $\mathrm{ACh}, \mathrm{AChE}$, and choline acetyltransferase (ChAT) in the hippocampus. To elucidate any anti-oxidative activities of TCE, we also ascertained the effects of TCE on reactive oxygen species (ROS), nitric oxide (NO), and malondialdehyde (MDA) in mice hippocampal tissue.

\section{Methods}

\section{Reagents}

Scopolamine hydrobromide, sodium nitrite (NaNO2), Griess reagent, dimethyl sulfoxide (DMSO), dichlorofluorescin diacetate (DCFDA), phosphate buffer, DPPH, donepezil, and ascorbic acid were purchased from Sigma-Aldrich (St. Louis, MO, USA). Anti-ChAT and glyceraldehyde 3-phosphate dehydrogenase (GAPDH) were purchased from EMD Millipore (Billerica, MA, USA). Anti-AChE was obtained from Abcam (Cambridge, MA, USA). Radioimmunoprecipitation assay (RIPA) buffer was obtained from Thermo Scientific (Waltham, MA, USA). All horseradish peroxidaseconjugated secondary antibodies were purchased from Santa Cruz (Santa Cruz, CA, USA). Chebulic acid, gallic acid, corilagin, chebulanin, 1,3,6-tri-O-galloyl $\beta$-Dglucose, chebulagic acid, and chebulinic acid were provided by Professor Sang Hyun Sung (Seoul National University, College of Pharmacy, South Korea). The purity of all reference chemical substances was higher than
95\%. HPLC-grade water and acetonitrile were purchased from J.T. Baker (Phillipsburg, NJ, USA).

\section{Plant materials and extraction}

Terminalia chebula Retz. is an accepted plant name and is listed in The Plant List (www.theplantlist.org). The ripe fruit of Terminalia chebula were purchased from Kwangmyungdang Medicinal Herbs Co. (Ulsan, South Korea) and identified by Dr. Go Ya Choi from the Kherb Research Center (Korea Institute of Oriental Medicine, Daejeon, South Korea). A voucher specimen (KIOM-Tech5) was deposited at the Convergence Research Center for Diagnosis, Treatment and Care System of Dementia (Korea Institute of Science and Technology, Seoul, South Korea).

The ripe fruit of Terminalia chebula (1.0 kg) were ground and soaked in $70 \% \mathrm{EtOH}(10 \mathrm{~L}$ for $15 \mathrm{~h})$ at $40^{\circ}$ C. The $70 \% \mathrm{EtOH}$ extracts were filtered, concentrated $\left(40{ }^{\circ} \mathrm{C}\right.$, EYELA rotary evaporation system, Tokyo Rikakikai, Tokyo, Japan), and dried (PVTFD-100 freeze drier, Ilshinbiobase, Dongducheon, South Korea) to produce a powdered extract $(268.4 \mathrm{~g})$, which was stored at $-20{ }^{\circ} \mathrm{C}$.

\section{Animals}

A total of 56 eight-week-old male C57BL/6 N mice were obtained from Charles River Co (Gapyeong, South Korea). We housed four to five animals per cage at a controlled temperature $\left(23 \pm 2{ }^{\circ} \mathrm{C}\right)$ and humidity $(50 \pm 10 \%)$. The mice were kept on $12 \mathrm{~h} / 12 \mathrm{~h}$ light/dark cycle (light from 08:00 A.M.- 08:00 P.M.). The mice received food and water ad libitum. The institutional animal care and use committee of Korea Institute of Science and Technology approved all of the experimental protocols described in the present study (Permit number: 2016-068). All procedures for the animal study were conducted in accordance with ARRIVE guidelines, and every effort was made to alleviate the suffering of the animals.

\section{Pharmacological treatment}

After adaptation for one week, the mice were randomly divided into six groups: (1) vehicle + vehicle $(n=10)$; $(2)$ TCE $(200 \mathrm{mg} / \mathrm{kg})+$ vehicle (TCE per se; $n=6)$; (3) vehicle + scopolamine (scopolamine per se; $n=10$ ); (4) TCE $\quad(100 \mathrm{mg} / \mathrm{kg})+$ scopolamine $(n=10) ; \quad(5) \quad$ TCE $(200 \mathrm{mg} / \mathrm{kg})+$ scopolamine $(n=10)$; and (6) donepezil $(5 \mathrm{mg} / \mathrm{kg})+$ scopolamine $(n=10)$. The doses of TCE were determined based on previous report [15]. We used saline for the vehicle group; TCE was therefore suspended in saline. The mice were orally administered saline, donepezil, or TCE on days $1-7$ to adapt them to the oral gavage and to adapt their metabolism prior to behavior tasks; treatment continued on days 8-14. Scopolamine $(1 \mathrm{mg} / \mathrm{kg})$ was dissolved in saline and treated intraperitoneally for 7 days (days 8-14). On days 8-14, 
TCE and scopolamine were administered at $60 \mathrm{~min}$ and 30 min before training, respectively.

\section{Morris water maze task}

Spatial learning and memory was evaluated using the water maze task as described in a previous study with minor modifications [16]. All mice underwent behavioral testing $30 \mathrm{~min}$ after scopolamine injection. The water maze comprised of a white circular tank $(183 \mathrm{~cm}$ diameter and $58 \mathrm{~cm}$ height) with a circular platform $(20 \mathrm{~cm}$ diameter). The tank was filled with water $\left(25 \pm 1{ }^{\circ} \mathrm{C}\right)$ and a nontoxic white dye. The platform hidden $0.5 \mathrm{~cm}$ beneath the water surface in one of tank's quadrants. The maze was enveloped in white curtains on which black patterns were placed as visual cues. All data were monitored using a camera recorder and analyzed using a video tracking system (HVS Image, Hampton, UK).

On days $8-13$, the mice underwent four training trials. If the mouse found the escape platform within $60 \mathrm{~s}$, the animal was left on the platform for an additional $20 \mathrm{~s}$. If the mouse did not find the platform within $60 \mathrm{~s}$, the animal was placed on the platform and permitted to remain there for $20 \mathrm{~s}$. The starting positions of the mice were changed across trials. Search errors were used as a metric for performance accuracy during training trials: the distance of the mouse from the platform was sampled 10 times per second, averaged across $1 \mathrm{~s}$, and its deviation from the most direct search path was calculated [17]; this method allowed us to remove a time bias associated with the repositioning of the platform. On days 11 and 13, all mice were subjected to probe tests without a platform for a $30 \mathrm{~min}$ interval between the last training trial and the probe trial to examine the retention of spatial memory. The average swimming speed of each mouse and their time spent in the target quadrant was measured.

\section{Sacrifice of mice}

On day 14, all mice were sacrificed 30 min after injection of scopolamine or the vehicle; cervical dislocation was performed before decapitation to provide each animal with a quick and painless death. The hippocampus was placed on ice and stored at $-80{ }^{\circ} \mathrm{C}$ until further biochemical analyses. All the mice were handled according to the animal welfare guidelines issued by Korean $\mathrm{Na}$ tional Institute of Health. The experimental scheme is depicted in Fig. 1.

\section{Determination of ex vivo $\mathrm{ACh}$ and $\mathrm{AChE}$ activity}

The activities of hippocampal ACh and AChE were determined using commercially available ACh quantitation and $\mathrm{AChE}$ activity assay kits according to the manufacturer's instructions (Sigma). The absorbance of ACh and AChE were measured using a microplate reader at $570 \mathrm{~nm}$ and $412 \mathrm{~nm}$, respectively (Bio Tek, Winooski, VT, USA). The values were reported as percentages of the vehicle control (vehicle + vehicle).

\section{Western blot analysis}

Sample preparation and western blotting were performed according to a previous report with minor modifications [16]. The tissues were homogenized in an RIPA buffer containing protease and phosphatase inhibitors (GenDEPOT, Barker, TX, USA). They were then centrifuged at $14,000 \times \mathrm{g}$ for $1 \mathrm{~h}$ at $4{ }^{\circ} \mathrm{C}$. The supernatants were collected, and Bradford assays were performed to determine protein concentrations. The proteins $(30 \mu \mathrm{g})$ were separated using SDS-PAGE and subsequently transferred to PVDF membranes. After $1 \mathrm{~h}$ of incubation in $5 \%$ fat-free dry milk, the membranes were incubated overnight with primary antibodies against ChAT (1: 1000), AChE (1:1000), and GAPDH (1:5000) at $4{ }^{\circ} \mathrm{C}$. The bands were normalized to GAPDH and quantified using an Image Gauge program (Fujifilm, Tokyo, Japan).

\section{Determination of ROS and NO levels}

ROS levels were determined according to a previous report [18]. Hippocampal homogenates were incubated with $15 \mu \mathrm{l}$ of $1 \mathrm{mM}$ DCFDA in the dark at $37^{\circ} \mathrm{C}$ for $1 \mathrm{~h}$. The fluorescence intensity was measured using a fluorescence microplate reader (Spectramax M5, Molecular Devices, Pennsylvania, USA) with an excitation and emission of 488 and $520 \mathrm{~nm}$, respectively. The values

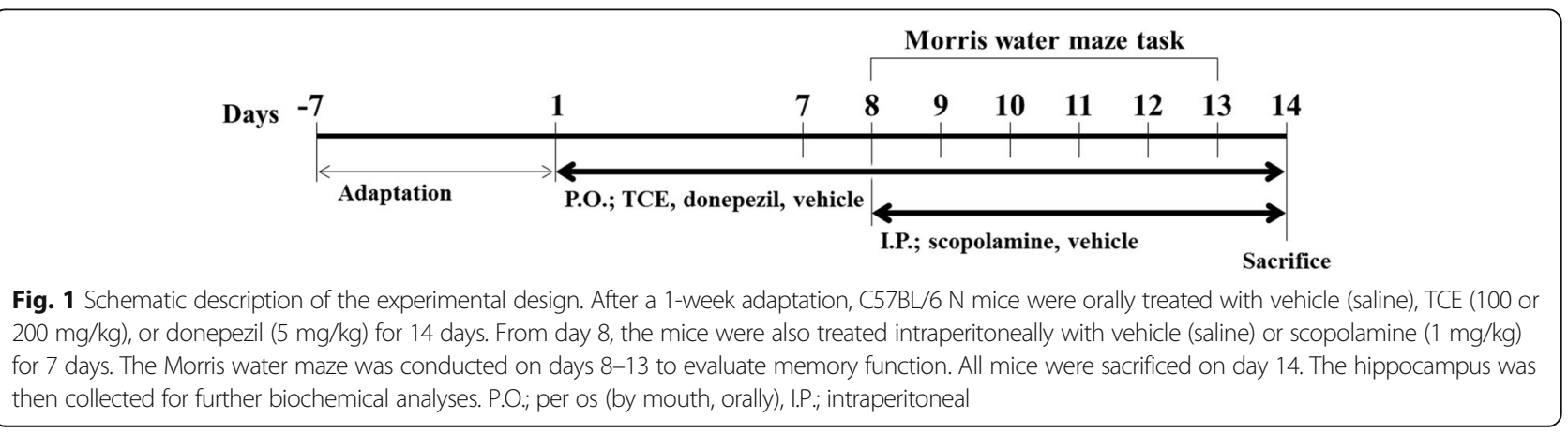


were reported as a percentage of the vehicle control (vehicle + vehicle).

NO production in the hippocampus was measured using the Griess method [19]. We incubated $40 \mu \mathrm{l}$ of the sample with $160 \mu \mathrm{l}$ of the Griess reagent in the dark at room temperature for $15 \mathrm{~min} . \mathrm{NaNO}_{2}$ was used to generate a standard nitrite concentration curve. The purple Azo dye product was detected at a wavelength of $540 \mathrm{~nm}$. The amount of $\mathrm{NO}$ was expressed as $\mu$ mole per mg protein.

\section{Estimation of lipid peroxidation}

MDA has been widely used to assess lipid peroxidation. The MDA concentration in the hippocampus was estimated using a commercial lipid peroxidation (MDA) assay kit according to the manufacturer's instructions (Abcam). Its absorbance was measured using a microplate reader at $532 \mathrm{~nm}$. The concentration of MDA was calculated using a reference standard. The results were expressed as nmole per mg protein.

High-performance liquid chromatography (HPLC) analyses HPLC analysis was performed using a Thermo Scientific Dionex Ultimate 3000 system. TCE and a mixed standard solution were separated using an YMC Triart C18 column $(4.6 \mathrm{~mm} \times 250 \mathrm{~mm}, 5.0 \mu \mathrm{m})$; the temperature of the column was maintained at $30{ }^{\circ} \mathrm{C}$ during the process. The mobile phases comprised of $0.1 \%(v / v)$ formic acid in water (solvent A) and acetonitrile (solvent $\mathrm{B}$ ). The following gradient system was used: $(B)=3 \%-30 \%(0-30 \mathrm{~min})$ and $30-$ 90\% (30-35 min). System re-equilibration lasted $10 \mathrm{~min}$. Analysis was performed at a flow rate of $1.0 \mathrm{~mL} / \mathrm{min}$ with UV detection at $280 \mathrm{~nm}$. The injection volume was $2 \mu \mathrm{l}$. All analyses were made in triplicate.

\section{Statistical analysis}

All data were expressed as the means \pm standard error of the mean (S.E.M). Statistical analysis was performed using SPSS software (SPSS Inc., Chicago, IL, USA). The search error data from the behavioral test were analyzed using two-way repeated analysis of variance (ANOVA). The correlation between obtained data was analyzed by the Pearson test. Other data were analyzed using one-way ANOVA. Post hoc analyses were subsequently performed using the least significant difference (LSD) test for comparison among experimental groups. The statistical significance was defined as $p$ values of less than 0.05 .

\section{Results}

Effect of TCE on learning and memory improvement in the Morris water maze

All experimental groups showed no toxicity in terms of general behavioral changes or mortality, and no adverse events were observed. Mouse body weights were measured on days 1 through 14 during treatment with TCE, donepezil, and scopolamine; no significant differences were found among the different groups (data not shown).

The Morris water maze test was performed to determine whether TCE attenuates learning and memory impairment induced by scopolamine. Two-way repeated ANOVA for search error revealed significant between-group treatment effects $\left(\mathrm{F}_{(5,50)}=15.468, p<0.001\right)$, training effects $\left(\mathrm{F}_{(5,250)}=\right.$ 78.640, $p<0.001)$, and treatment $\times$ training interaction effects $\left(\mathrm{F}_{(25250)}=3.310, p<0.001\right)$. As shown in Fig. 2a, scopolamine per se treatment showed a higher search error than $\mathrm{VEH}+\mathrm{VEH}$ control during the 6 days of training; this result suggests that scopolamine triggered learning deficits $(p<0.001)$. However, the scopolamine-treated mice that received either 100 or $200 \mathrm{mg} / \mathrm{kg}$ of TCE performed significantly better than those that received scopolamine alone, indicating that learning impairment was attenuated following TCE treatment $(p<0.05$ and $p<0.001$, respectively). Mice treated with donepezil (5 mg/kg), a well-known reversible AChE inhibitor used as a positive control, showed better performance than mice injected with scopolamine alone $(p$ $<0.001)$. There was no difference in TCE per se-treated mice compared to vehicle-treated mice. In addition, the swimming speed did not differ among the groups, indicating that the locomotor activity of mice was not affected by scopolamine, TCE, or donepezil (Fig. 2b, $\mathrm{F}_{(5,50)}$ $=0.766, p=0.578$ ). We also measured the body weights of all mice during drug treatment and behavioral tasks, but there was no significant difference among the six groups (data not shown).

The mice underwent two probe trials to determine memory retention on days 11 (1st probe) and 13 (2nd probe). In the 1 st probe test, one-way ANOVA revealed significant differences between-group effects $\left(\mathrm{F}_{(5,50)}=9.568, p<0.001\right)$. Post hoc analyses showed that the time to the target quadrant of the scopolamine per se-treated group was significantly lower than that of the vehicle control (Fig. 2c; $p<0.001$ ). Treatment with TCE $(200 \mathrm{mg} / \mathrm{kg})$, however, significantly increased the time to the target quadrant $(p<0.05)$. Donepezil showed effects similar to those of TCE $(200 \mathrm{mg} / \mathrm{kg})$ treatment $(p<0.05)$. In addition, there was no difference between the TCE per se-treated mice and the vehicle control.

In the 2nd probe test, one-way ANOVA revealed significant between-group effects $\left(\mathrm{F}_{(5,50)}=5.411, p<0.001\right)$. Similar to the results from the 1 st probe, there was a significant difference between the $\mathrm{VEH}+\mathrm{VEH}$ and $\mathrm{VEH}+$ SCO groups $(p<0.001)$. Though TCE-treated mice spent more time in the target quadrant than scopolamine per se group, the effect was nonsignificant. The swimming routes further confirmed that TCE-treated mice remained in the target quadrant longer than did the scopolamine per se treated mice (Fig. 2d). Taken together, these results suggest that TCE ameliorated 


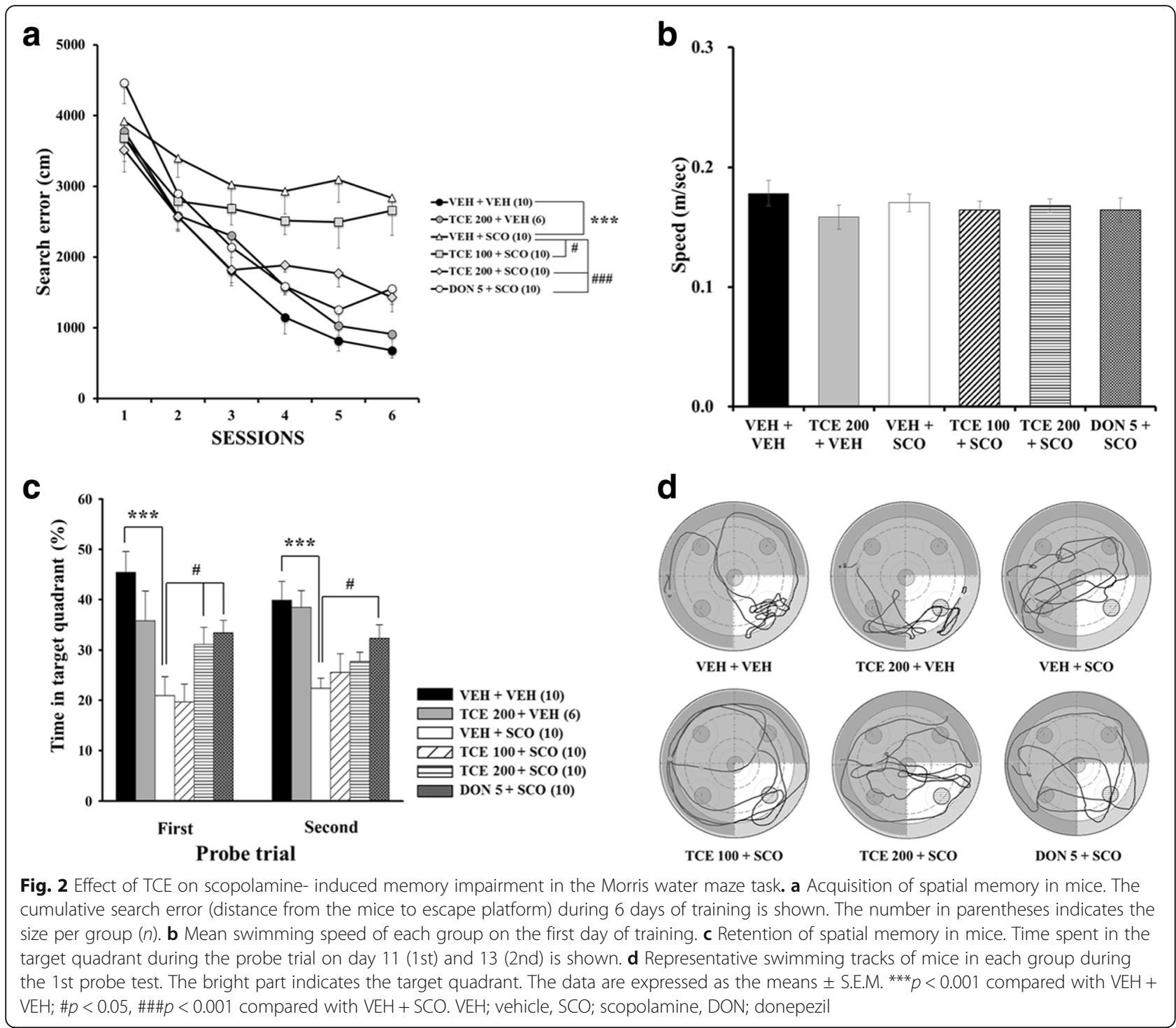

induced cognitive impairments related to spatial learning and memory.

\section{Effect of TCE on cholinergic system in the hippocampus}

To investigate whether TCE administration had an effect on cholinergic pathways, ACh levels in the hippocampus was measured. One-way ANOVA indicated significant between-group effects $\left(\mathrm{F}_{(5,17)}=4.626\right.$, $p=0.014)$. As shown in Fig. 3a, scopolamine per setreated mice significantly reduced hippocampal ACh levels in the hippocampus relative to vehicle controls $(p<0.01)$. Treatment with TCE $(200 \mathrm{mg} / \mathrm{kg})$ induced a significant increase in ACh levels when compared with the $\mathrm{VEH}+\mathrm{SCO}$ group $(p<0.05)$. Donepezil showed a significant increase in ACh levels relative to vehicle controls $(p<0.05)$; this effect was absent when vehicle controls were compared with the TCE per se treated mice compared to vehicle control (Fig. 3a).

We also measured hippocampal AChE activity to elucidate the underlying mechanism of a possible TCEinduced increase of ACh levels. One-way ANOVA indicated a statistically significant between-group effects $\left(\mathrm{F}_{(5,29)}=10.999, p<0.001\right)$. In the scopolamine-injected group, a significant increase in AChE activity was observed in the hippocampus (Fig. 3b; $p<0.001$ ). TCE administration inhibited AChE activation induced by scopolamine treatment in a dose-dependent manner (Fig. 3b; $p<0.01$ for $100 \mathrm{mg} / \mathrm{kg}$ and $p<0.001$ for $200 \mathrm{mg} / \mathrm{kg}$ ). Donepezil also resulted in significant inhibition of AChE activity $(p<0.001)$. The difference in AChE levels between the TCE per se treatment and vehicle treatment were nonsignificant. 

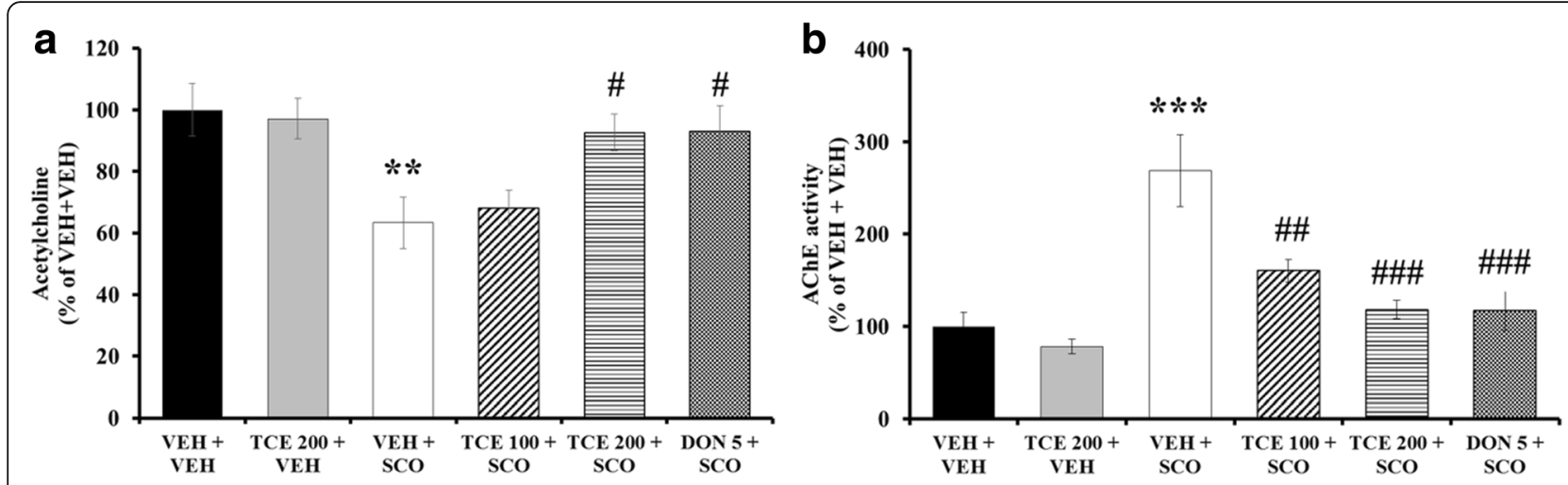

Fig. 3 Effect of TCE on hippocampal ACh levels and AChE activity in the scopolamine-induced model. a The level of ACh in hippocampal tissue is shown ( $n=3$ per group). b Hippocampal AChE levels are presented ( $n=5$ per group). Bar graph is expressed as a percentage of VEH $+V E H$ control. The data are expressed as the means \pm S.E.M. ${ }^{*} p<0.05,{ }^{* * *} p<0.001$ compared with VEH + VEH; \#p $<0.05, \# \# p<0.01$, \#\#\#p $<0.001$ compared with VEH + SCO. VEH; vehicle, SCO; scopolamine, DON; donepezil

To examine whether TCE treatment influenced protein expression associated with the cholinergic pathway, we performed western blotting for ChAT and AChE on hippocampal tissue (Fig. 4a). One-way ANOVA revealed between-group effects $\left(\mathrm{F}_{(5,29)}=5.276, p=0.002\right)$ in ChAT expression. Consistent with a previous report [16], there were no significant differences between vehicle control and scopolamine per se-treated mice (Fig. 4b). Treatment with scopolamine and TCE at a dose of $200 \mathrm{mg} / \mathrm{kg}$ significantly up-regulated the expression of ChAT in the hippocampus (Fig. 4b; $p<0.05$ ). Donepezil also showed significantly increased ChAT expression $(p<0.01)$. Moreover, one-way ANOVA indicated between-group changes in the $\mathrm{AChE}$ expression levels in the hippocampus $\left(\mathrm{F}_{(5,29)}\right.$ $=3.882, p=0.010)$ : TCE at $200 \mathrm{mg} / \mathrm{kg}$ reversed the scopolamine-mediated increase of AChE (Fig. 4c; $p<0.01$ ) and Donepezil induced a significant decrease in AChE expression $(p<0.001)$. TCE per se treatment did not exhibit any significant changes when compared with vehicle treatment alone. These findings suggest that TCE may

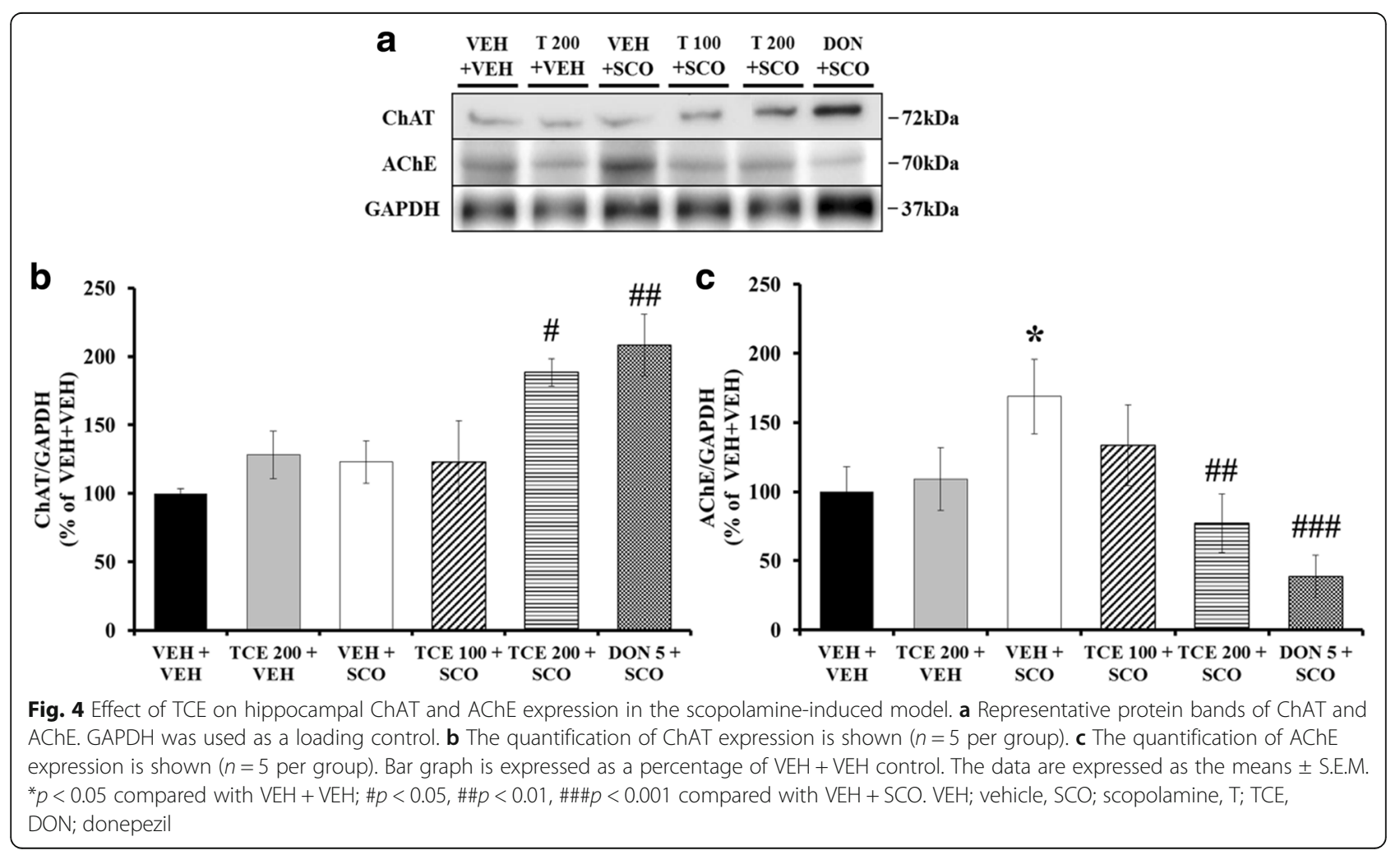


protect against scopolamine-induced memory impairment through mechanisms related to cholinergic function.

\section{Effect of TCE on oxidative damage in the hippocampus}

We investigated ROS, NO, and MDA levels in hippocampal tissue to determine whether TCE inhibits oxidative damage induced by scopolamine. Regarding the effect of TCE on the ROS production, one-way ANOVA revealed significant between-group effects $\left(\mathrm{F}_{(5,17)}=8.592, p<0.001\right)$. The ROS level was significantly increased in scopolamine-treated mice relative to vehicle-treated mice $(p<0.001)$; this elevation was significantly attenuated after treatment with either TCE at $200 \mathrm{mg} / \mathrm{kg}$ or donepezil (Fig. 5a; $p<0.01$ ). One-way ANOVA revealed significant between-group effects on NO production $\left(\mathrm{F}_{(5,17)}=10.631, p<0.001\right)$. Scopolamine administration notably up-regulated NO levels in the hippocampus $(p<0.001)$. Administration of TCE at $100 \mathrm{mg} / \mathrm{kg}$ induced a nonsignificant attenuation of NO levels, while the $200 \mathrm{mg} /$ $\mathrm{kg}$ dosage induced a significant mitigation (Fig. 5b; $p<0.01$ ). Donepezil also resulted in the significant reduction of $\mathrm{NO}$ concentration $(p<0.001)$. MDA levels in the hippocampus were also measured to determine the effect of TCE on lipid peroxidation; One-way ANOVA indicated significant changes between groups $\left(\mathrm{F}_{(5,17)}=43.445, p<0.001\right)$. Compared with the vehicle treatment group, scopolamine treatment generated a significant increase in MDA concentrations $(p<0.001)$. TCE treatment $(100$ and $200 \mathrm{mg} /$ $\mathrm{kg}$ ) affected a significant decrease of MDA levels relative to scopolamine treatment (Fig. 5c; $p<0.05$ and $p<0.001$, respectively). A reduced MDA concentration was also observed in the donepezil treatment group $(p<0.001)$. These results suggest that TCE effectively attenuated oxidative damage induced by scopolamine.

\section{HPLC analysis of TCE}

The HPLC chromatogram characteristics of the seven compounds in TCE (chebulic acid, gallic acid, corilagin, chebulanin, 1,3,6-tri-O-galloyl $\beta$-D-glucose, chebulagic acid, and chebulinic acid), including the retention time and UV spectrum, were determined. The retention times for the seven standards of chebulic acid, gallic acid, corilagin, chebulanin, 1,3,6-tri-O-galloyl $\beta$-D-glucose, chebulagic acid, and chebulinic acid were 5.69, 9.60, 20.34, 20. 76, 22.43, 24.75, and 28.04 min, respectively (Fig. 6 and Table 1). All of the calibration curves for the seven compounds exhibited good linearity $\left(r^{2}>0.9990\right)$. Table 1

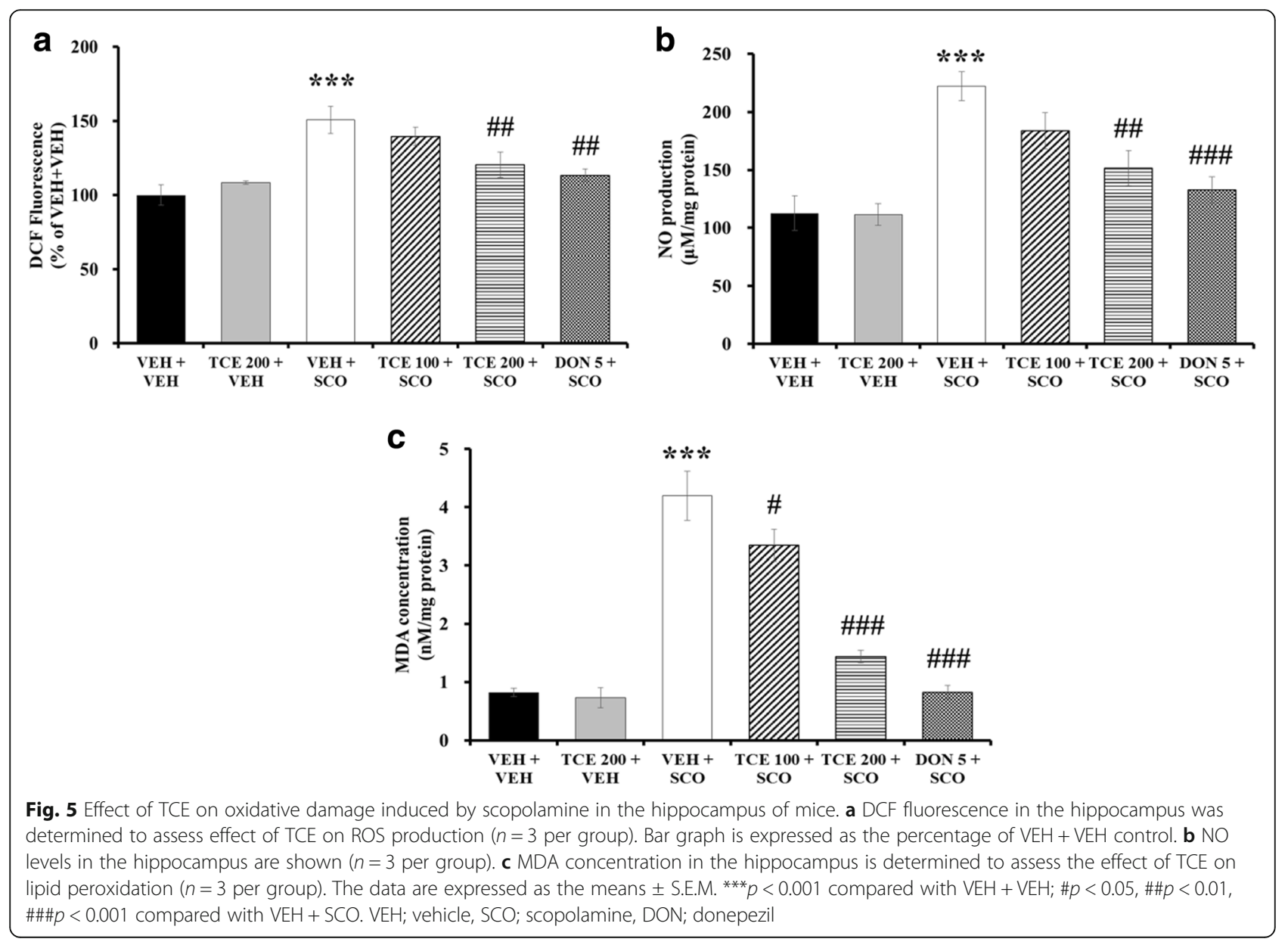




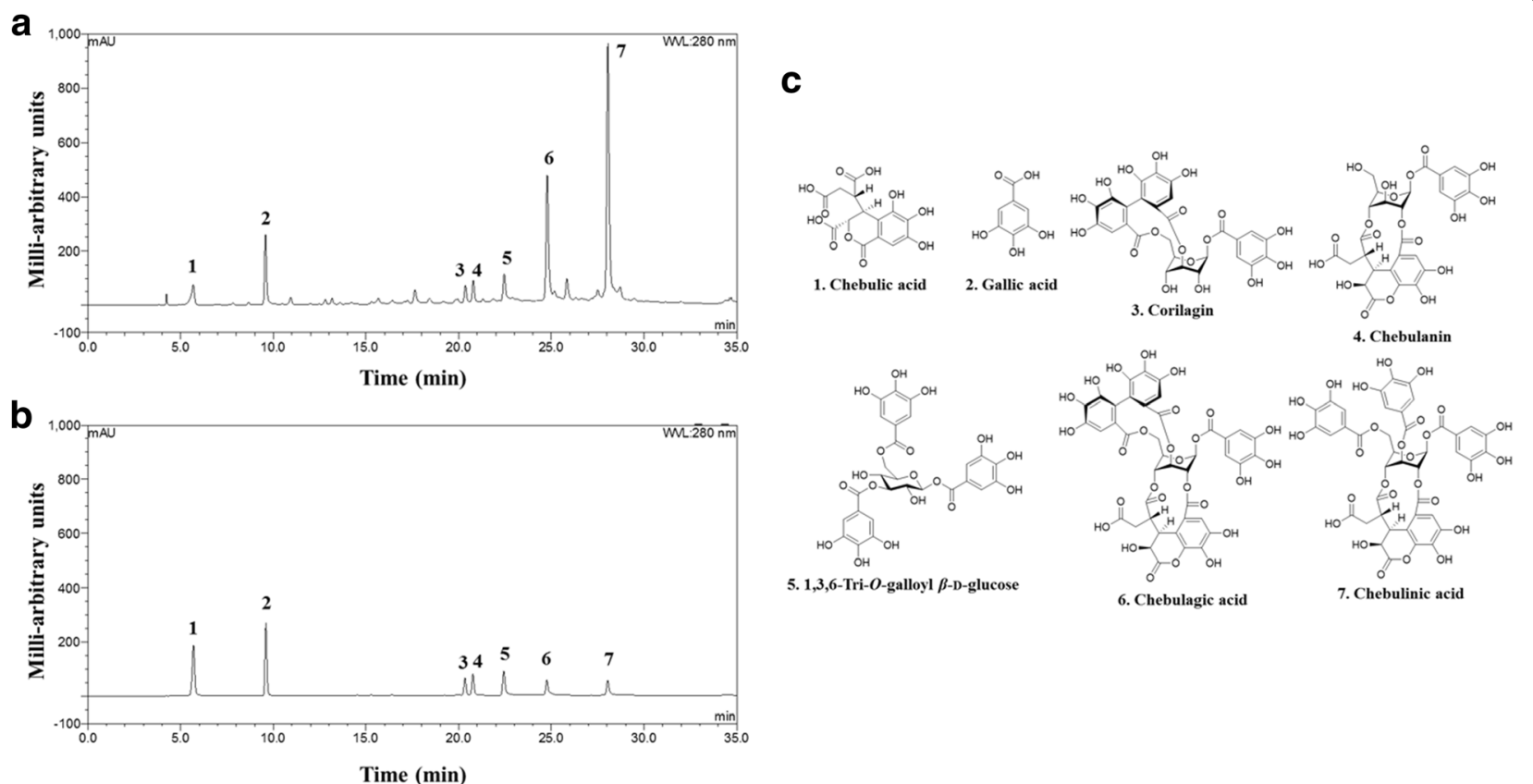

Fig. 6 Representative HPLC-UV chromatogram. a HPLC-UV chromatogram of TCE. b HPLC-UV chromatogram of seven standard compounds in the mixed solution. c Chemical structures of the seven main compounds in TCE

shows that chebulagic acid $(169.7 \mathrm{mg} / \mathrm{g})$ and chebulinic acid $(263.7 \mathrm{mg} / \mathrm{g})$ are the principal components of TCE.

\section{Discussion}

Terminalia chebula, the main material examined in the present study, is widely used as a medicinal herb to treat diabetes, asthma, sore throat, and other conditions [20]. Much research has already been conducted to investigate its activity and applications; however, the antiAChE and anti-oxidative effects of Terminalia chebula have primarily been observed in vitro. Though one study previously reported nootropic effects of TCE in normal mice in vivo [21], whether TCE could alleviate amnesia following cholinergic blockade remains unknown. The present study therefore examine the anti-amnesic effect of TCE using mice treated with scopolamine, an inhibitor of central cholinergic transmission. Based on findings from previous reports, we administered TCE at 100 and

Table 1 Proportion of the seven TCE constituent compounds ${ }^{a}$

\begin{tabular}{lll}
\hline No. & Analytes & Content (\%) \\
\hline 1 & Chebulic acid & 1.60 \\
2 & Gallic acid & 2.85 \\
3 & Corilagin & 2.76 \\
4 & Chebulanin & 2.56 \\
5 & $1,3,6-$-Tri-O-galloyl $\beta$-D-glucose & 2.82 \\
6 & Chebulagic acid & 16.97 \\
7 & Chebulinic acid & 26.37 \\
\hline
\end{tabular}

${ }^{\mathrm{a}}$ All analyses were conducted in triplicate
$200 \mathrm{mg} / \mathrm{kg}$ doses $[15,21]$. As confirmed by the results the water maze trials, the findings demonstrate that TCE has an anti-amnesic effect on scopolamine-treated mice. We also observed the anti-AChE and anti-oxidative effects of TCE using several assays, including ex vivo experiments. In this respect, Terminalia chebula could potentially represent an important alternative drug for addressing amnesia.

In the present study, we confirmed that TCE is composed of seven compounds and ascertained their quantitative differences through an HPLC analysis. Most of the compounds found in TCE are phenolic, including hydrolyzable tannins. We recently reported that chebulic acid and chebulanin showed AChE inhibitory activity at a concentration of $10 \mu \mathrm{M}$. In particular, chebulanin showed the most potent AChE inhibitory activity, with an $\mathrm{IC}_{50}$ of $21.36 \mu \mathrm{M}$ [22]. Considering the anti-oxidative effects of both compounds as reported in previous studies $[23,24]$, chebulanin and chebulic acid may be the major anti-amnesic constituents of TCE. Future in vivo studies are required to determine the utility of these compounds in developing pharmacological agents.

ACh signaling plays a crucial role in maintaining memory function [25]. Since patients with memory disorders typically exhibit cholinergic deficits (i.e., abnormally elevated AChE activity and reduced ACh levels), many studies have focused on the restoration of the cholinergic systems to improve memory [26]. According to a previous report, scopolamine causes memory impairments by means of affecting cholinergic 
pathways [27]; the scopolamine model used in the present study is therefore suitable for assessing the effectiveness of anti-amnesic agents.

In the present investigation, all mice underwent analysis using the Morris water maze to evaluate learning and memory function. The water maze serves as a powerful and sensitive tool for assessing hippocampal-dependent spatial learning and memory [28]. We conducted a cumulative search error indicating the distance of each mouse from the platform during daily training sessions to characterize spatial learning,; the values thus reflect both proximity and latency [17]. The results demonstrated that TCE treatment improved search error during training trials, suggesting the amelioration of learning impairment. Moreover, the probe test revealed that TCE administration at a dose of $200 \mathrm{mg} / \mathrm{kg}$ elevated the time spent in the target quadrant; the results thus evince the efficacy of TCE in improving memory retention. Further study is needed to investigate whether TCE might affect the retrieval of memory, another stage of the memory process.

Our biochemical analyses further demonstrated that TCE exhibits therapeutic effects on cholinergic dysfunction in the hippocampus: a major region of the brain involved in learning and memory function, particularly in spatial memory [29]. Many pathological changes to brain structure and function have been detected in the hippocampus of patients with amnesia; specifically, abnormalities in the cholinergic system were observed in the hippocampus of amnesia patients [26]. It has also been reported that the hippocampus is vulnerable to damage after scopolamine administration [30]. In this context, TCE administration mitigated AChE activity and expression in hippocampal tissue. We also found a negative correlation between AChE activity and the time spent in the target quadrant (Additional file 1: Figure S1). Although ChAT expression was not affected by scopolamine administration, increased ChAT expression was observed in the scopolamine + TCE $(200 \mathrm{mg} / \mathrm{kg})$ group. We did not, however, observe significant differences in hippocampal ChAT expression between the vehicle control and TCE per se-treated mice. Further studies are required to reveal the cause of these differences or lack thereof. Taken together, these results suggest that the regulation of the cholinergic system may underlie the anti-amnesic effects of TCE.

Increase in oxidative stress with age is the main risk factor for amnesia; the excessive generation of oxidative products, including ROS, contributes to the disturbance of calcium homeostasis, increased neurotoxic activity, and subsequent elevated lipid peroxidation [31]. Delaying or preventing oxidative damage using anti-oxidants may therefore present a promising therapeutic strategy for treating amnesia. We investigated the anti-oxidative effects of TCE using an in vivo scopolamine model as this model encompasses notable oxidative damage. In our study, increased ROS and MDA concentrations were correlated with memory impairment (Additional file 1: Figure S1). The results showed that TCE treatment attenuated up-regulated levels of ROS, NO, and MDA induced by scopolamine in the hippocampal region, which is vulnerable to oxidative stress [32]. TCE treatment may thus alleviate amnesic symptoms by exerting antioxidative effects.

A previous study has reported that levels of AChE splice variants were increased under conditions of oxidative-stress [33]. We therefore analyzed the correlation between AChE activity and oxidative markers and observed a significant correlation between AChE activity and ROS, as well as between AChE activity and MDA (Additional file 2: Figure S2); our findings agree with those of a previous study [34]. These results therefore suggest a possible interaction between scopolamine-induced cholinergic modulation and oxidative stress. However, further research is needed to clarify the mechanism underlying the interaction.

Our study has several limitations. First, we restricted our investigation to spatial learning and memory. The effect of TCE on other types of learning and memory (e.g., object recognition memory and fear memory) remains unknown. Second, we did not investigate which channel(s) or receptor(s) are involved in cholinergic transmission after the administration of TCE. Lastly, we do not know whether TCE has a beneficial effect on amnesia in chronic neurodegenerative diseases, such as Alzheimer's disease (AD). Further studies are therefore necessary to explore the anti-amnesic effects of TCE using a transgenic animal model of AD.

\section{Conclusion}

The present study was conducted to evaluate the antiamnesic activity of TCE in a scopolamine-induced murine model of memory impairment. Our data demonstrated that TCE administration ameliorated cognitive deficits measured by performance in water maze tasks. TCE treatment reduced $\mathrm{AChE}$ and increased ChAT expression, thus elevating ACh levels in hippocampal tissue of scopolamine-induced mice. Furthermore, decreases ROS, NO, and MDA levels in the hippocampus evince the anti-oxidative capability of TCE treatment. The results indicate that the underlying mechanism of learning and memory improvement may involve modulations of the cholinergic system and the reduction of oxidative stress. These findings thus provide evidence for the potential of TCE extract as a natural, alternative treatment for amnesia. 


\section{Additional files}

Additional file 1: Figure S1. Correlation graph between behavior test and measured biomarkers. (A) Correlation of time in target quadrant of the first probe test (\%) with AChE activity (\% of VEH + VEH). (B) Correlation of time in target quadrant of the first probe test (\%) with DCF fluorescence (\% of $\mathrm{VEH}+\mathrm{VEH}$ ). (C) Correlation of time in target quadrant of the first probe test (\%) with MDA concentration (nM/mg protein). (TIF $1767 \mathrm{~kb}$ )

Additional file 2: Figure S2. Correlation graph between AChE activity and oxidative markers. (A) Correlation of AChE activity (\% of VEH + VEH) with DCF fluorescence (\% of VEH + VEH). (B) Correlation of AChE activity (\% of $\mathrm{VEH}+\mathrm{VEH}$ ) with MDA concentration (nM/mg protein). (TIF $1099 \mathrm{~kb}$ )

\section{Abbreviations}

ACh: Acetylcholine; AChE: Acetylcholinesterase; AD: Alzheimer's disease; ChAT: Choline acetyltransferase; DCFDA: Dichlorofluorescin diacetate; DMSO: Dimethyl sulfoxide; GAPDH: Glyceraldehyde 3-phosphate dehydrogenase; HPLC: High-performance liquid chromatography; MDA: Malondialdehyde; NO: Nitric oxide; ROS: Reactive oxygen species; TCE: Terminalia chebula extract

\section{Acknowledgments}

This work was financially supported by grants from the National Research Council of Science and Technology (NST) by the Korean government (MSIP) (CRC-15-04-KIST, G15120, G16230, K16850 and K17850).

\section{Availability of data and materials}

The datasets used and/or analysed during the current study available from the corresponding author on reasonable request.

\section{Authors' contributions}

MSK and WKJ designed the study and wrote the manuscript. MSK, DYL, JL, and HWK performed the experiments. SHS and JSH conducted the statistical analysis and interpretation of the data. All authors read and approved the final manuscript.

\section{Ethics approval}

The institutional animal care and use committee of Korea Institute of Science and Technology approved all of the experimental protocols described in the present study (Permit number: 2016-068). All procedures for the animal study were conducted in accordance with ARRIVE guidelines.

\section{Competing interests}

The authors declare that there is no competing interests.

\section{Publisher's Note}

Springer Nature remains neutral with regard to jurisdictional claims in published maps and institutional affiliations.

\begin{abstract}
Author details
${ }^{1}$ Herbal Medicine Research Division, Korea Institute of Oriental Medicine, Daejeon 34054, South Korea. ${ }^{2}$ Convergence Research Center for Diagnosis, Treatment and Care System of Dementia, Korea Institute of Science and Technology, Seoul 02792, South Korea. ${ }^{3}$ College of Pharmacy, Seoul National University, Seoul 08826, South Korea. ${ }^{4}$ Department of Biological Sciences, Konkuk University, Seoul 05029, South Korea.
\end{abstract}

\section{Received: 25 July 2017 Accepted: 18 April 2018}

\section{Published online: 02 May 2018}

\section{References}

1. Wimo A, Jönsson L, Bond J, Prince M, Winblad B, International AD. The worldwide economic impact of dementia 2010. Alzheimers Dement. 2013. $9(1): 1-11$.

2. Hasselmo ME. The role of acetylcholine in learning and memory. Curr Opin Neurobiol. 2006;16(6):710-5.

3. Upadhyaya P, Seth V, Ahmad M. Therapy of Alzheimers disease: an update. Afr J Pharm Pharmacol. 2010;4(6):408-21.
4. Kumar A, Singh A. A review on Alzheimer's disease pathophysiology and its management: an update. Pharmacol Rep. 2015;67(2):195-203.

5. Rountree SD, Chan W, Pavlik VN, Darby EJ, Siddiqui S, Doody RS. Persistent treatment with cholinesterase inhibitors and/or memantine slows clinical progression of Alzheimer disease. Alzheimers Res Ther. 2009;1(2):1.

6. Dastmalchi K, Dorman HD, Vuorela H, Hiltunen R. Plants as potential sources for drug development against Alzheimer's disease. Int J Biomed Pharm Sci. 2007;1(2):83-104.

7. Rao NK, Nammi S. Antidiabetic and renoprotective effects of the chloroform extract of Terminalia chebula Retz. seeds in streptozotocin-induced diabetic rats. BMC Complement Altern Med. 2006;6(1):1.

8. Aher $\mathrm{V}$, Wahi A. Immunomodulatory activity of alcohol extract of Terminalia chebula retz combretaceae. Trop J Pharm Res. 2011:10(5):567-75.

9. Sharma P, Prakash T, Kotresha D, Ansari MA, Sahrm UR, Kumar B, Debnath J, Goli D. Antiulcerogenic activity of Terminalia chebula fruit in experimentally induced ulcer in rats. Pharm Biol. 2011;49(3):262-8.

10. Afshari AR, Sadeghnia HR, Mollazadeh H. A review on potential mechanisms of Terminalia chebula in Alzheimer's disease. Adv Pharmacol Sci. 2016;2016: 8964849.

11. Gaire BP, Jamarkattel-Pandit N, Lee D, Song J, Kim JY, Park J, Jung S, Choi H-Y, Kim H. Terminalia chebula extract protects OGD-R induced PC12 cell death and inhibits LPS induced microglia activation. Molecules. 2013;18(3):3529-42.

12. Sancheti S, Um B-H, Seo S-Y. 1, 2, 3, 4, 6-penta-O-galloyl-B-D-glucose: a cholinesterase inhibitor from Terminalia chebula. S Afr J Bot. 2010;76(2):285-8.

13. Sulaiman C, Sadashiva C, George S, Balachandran I. Acetylcholinestrase inhibition and antioxidant activity of Terminalia chebula. Retz J Trop Med Plants. 2012;13(2):125-7.

14. Ahmed T, Gilani A-H. Inhibitory effect of curcuminoids on acetylcholinesterase activity and attenuation of scopolamine-induced amnesia may explain medicinal use of turmeric in Alzheimer's disease. Pharmacol Biochem Behav. 2009;91(4):554-9.

15. Sarkar R, Hazra B, Mandal N. Reducing power and iron chelating property of Terminalia chebula (Retz.) alleviates iron induced liver toxicity in mice. BMC Complement Altern Med. 2012;12(1):144.

16. Kim M-S, Jeon WK, Lee KW, Park YH, Han J-S. Ameliorating effects of ethano extract of Fructus mume on scopolamine-induced memory impairment in mice. Evid Based Compl Altern Med. 2015;2015:102734.

17. Gallagher M, Burwell R, Burchinal MR. Severity of spatial learning impairment in aging: development of a learning index for performance in the Morris water maze. Behav Neurosci. 1993;107(4):618.

18. Rizzetti DA, Altermann CDC, Martinez CS, Peçanha FM, Vassallo DV, Uranga-Ocio JA, Castro MM, Wiggers GA, Mello-Carpes PB. Ameliorative effects of egg white hydrolysate on recognition memory impairments associated with chronic exposure to low mercury concentration. Neurochem Int. 2016;101:30-7.

19. Green LC, Wagner DA, Glogowski J, Skipper PL, Wishnok JS, Tannenbaum SR. Analysis of nitrate, nitrite, and [15 N] nitrate in biological fluids. Anal Biochem. 1982;126(1):131-8.

20. Reddy DB, Reddy T, Jyotsna G, Sharan S, Priya N, Lakshmipathi V, Reddanna P. Chebulagic acid, a COX-LOX dual inhibitor isolated from the fruits of Terminalia chebula Retz., induces apoptosis in COLO-205 cell line. J Ethnopharmacol. 2009;124(3):506-12.

21. Rao N, Palaksha M, Satish S, Ravishankar S. The effects of ethanolic extract in dried fruits of Terminalia chebula on learning and memory in mice. Asian J Biomed Pharm Sci. 2013;3(20):59.

22. Kim M-S, Lee DY, Sung SH, Jeon WK. Anti-cholinesterase activities of hydrolysable tannins and polyhydroxytriterpenoid derivatives from Terminalia chebula Retz. Fruit Rec Nat Prod. 2018;12(3):284-9.

23. An J, Li T, Dong Y, Li Z, Huo J. Terminalia chebulanin attenuates psoriatic skin lesion via regulation of heme oxygenase-1. Cell Physiol Biochem. 2016: 39(2):531-43.

24. Lee H-S, Jung S-H, Yun B-S, Lee K-W. Isolation of chebulic acid from Terminalia chebula Retz. And its antioxidant effect in isolated rat hepatocytes. Arch Toxicol. 2007;81(3):211-8

25. Contestabile A. The history of the cholinergic hypothesis. Behav Brain Res. 2011;221(2):334-40.

26. Parent MB, Baxter MG. Septohippocampal acetylcholine: involved in but not necessary for learning and memory? Learn Mem. 2004;11(1):9-20.

27. Yang $W, Y u$ J, Zhao L, Ma N, Fang Y, Pei F, Mariga AM, Hu Q. Polysaccharides from Flammulina velutipes improve scopolamineinduced impairment of learning and memory of rats. J Funct Foods. 2015;18:411-22. 
28. Logue SF, Paylor R, Wehner JM. Hippocampal lesions cause learning deficits in inbred mice in the Morris water maze and conditioned-fear task. Behav Neurosci. 1997;111(1):104.

29. Broadbent NJ, Squire LR, Clark RE. Spatial memory, recognition memory, and the hippocampus. Proc Natl Acad Sci U S A. 2004;101(40):14515-20.

30. Lee B, Park J, Kwon S, Park MW, Oh SM, Yeom MJ, Shim I, Lee HJ, Hahm DH. Effect of wild ginseng on scopolamine-induced acetylcholine depletion in the rat hippocampus. J Pharm Pharmacol. 2010;62(2):263-71.

31. Yu SP, Canzoniero LM, Choi DW. Ion homeostasis and apoptosis. Curr Opin Cell Biol. 2001;13(4):405-11.

32. Mattson MP, Pedersen WA, Duan W, Culmsee C, Camandola S. Cellular and molecular mechanisms underlying perturbed energy metabolism and neuronal degeneration in Alzheimer's and Parkinson's diseases. Ann N Y Acad Sci. 1999;893(1):154-75.

33. Härtl R, Gleinich A, Zimmermann M. Dramatic increase in readthrough acetylcholinesterase in a cellular model of oxidative stress. J Neurochem. 2011;116(6):1088-96.

34. Rahimzadegan M, Soodi M. Comparison of memory impairment and oxidative stress following single or repeated doses administration of scopolamine in rat hippocampus. Basic Clin Neurosci. 2018;9(1):5-14.

Ready to submit your research? Choose BMC and benefit from:

- fast, convenient online submission

- thorough peer review by experienced researchers in your field

- rapid publication on acceptance

- support for research data, including large and complex data types

- gold Open Access which fosters wider collaboration and increased citations

- maximum visibility for your research: over $100 \mathrm{M}$ website views per year

At BMC, research is always in progress.

Learn more biomedcentral.com/submissions 